\title{
Properties of certain integer-valued analogues of Dedekind sums
}

by

Jeffrey L. Meyer (Syracuse, N.Y.)

1. Introduction. B. C. Berndt [2] demonstrated that certain sums, first examined by G. H. Hardy [6], [7, pp. 362-392] and arising in the transformation formulas of the logarithms of the classical theta-functions, are analogous to the regular Dedekind sum. In this paper, we apply $\mathrm{H}$. Rademacher's questions [12], [13, p. 112] about Dedekind sums to the study of these analogous sums. To introduce these sums, let $\Im(z)>0, q=e^{\pi i z}$,

$$
\theta(z)=\sum_{n=-\infty}^{\infty} q^{n^{2}} \text { and } \theta_{4}(z)=\sum_{n=-\infty}^{\infty}(-1)^{n} q^{n^{2}} .
$$

Let $V=\left[\begin{array}{ll}a & b \\ c & d\end{array}\right]$ and define $V z=(a z+b) /(c z+d)$. We also define the standard modular groups

$$
\begin{aligned}
\Gamma(1) & =\{V: a, b, c, d \in \mathbb{Z}, c>0 \text { with } a d-b c=1\}, \\
\Gamma_{\theta} & =\{V \in \Gamma(1): a \equiv d, b \equiv c(\bmod 2)\}, \\
\Gamma^{0}(2) & =\{V \in \Gamma(1): b \equiv 0(\bmod 2)\} .
\end{aligned}
$$

In [2], Berndt proved the following results. If $V \in \Gamma_{\theta}$, then

$$
\log \theta(V z)=\log \theta(z)+\frac{1}{2} \log (c z+d)-\frac{1}{4} \pi i+\frac{1}{4} \pi i S(d, c),
$$

where

$$
S(d, c)=\sum_{j=1}^{c-1}(-1)^{j+1+[d j / c]} .
$$

If $V \in \Gamma^{0}(2)$, then

$$
\log \theta_{4}(V z)=\log \theta_{4}(z)+\frac{1}{2} \log (c z+d)-\frac{1}{4} \pi i-\frac{1}{4} \pi i S_{4}(d, c),
$$

1991 Mathematics Subject Classification: 11F20, 11A99. 
where

$$
S_{4}(d, c)=\sum_{j=1}^{c-1}(-1)^{[d j / c]} .
$$

We define the empty sums $S(d, 1)=S_{4}(d, 1)=0$. In this paper, we examine properties of the sums $S(d, c)$ and $S_{4}(d, c)$ outside of the context of the transformation formulas. For results related to the transformation formulas, see L. A. Goldberg's thesis [5].

The most well-known property of the Dedekind sum $s(d, c)$ defined by

$$
s(d, c)=\sum_{j=1}^{c}\left(\left(\frac{j}{c}\right)\right)\left(\left(\frac{d j}{c}\right)\right)
$$

with

$$
((x))= \begin{cases}0 & \text { if } x \in \mathbb{Z}, \\ x-[x]-1 / 2 & \text { otherwise }\end{cases}
$$

is the reciprocity law, first proved by Dedekind [3]:

RECIPROCITY LAW FOR $s(d, c)$. If $(c, d)=1$ and $c, d>0$, then

$$
s(c, d)+s(d, c)=-\frac{1}{4}+\frac{d}{12 c}+\frac{c}{12 d}+\frac{1}{12 c d} .
$$

In [2], Berndt proves the reciprocity theorem for $S(d, c)$ :

RECIPROCITy LAW FOR $S(d, c)$. If $(c, d)=1, c+d$ is odd and $c, d>0$, then

$$
S(c, d)+S(d, c)=1 .
$$

There is no reciprocity formula for $S_{4}(d, c)$ in this same sense. (See [2] for so-called hybrid reciprocity formulas, and the author's thesis [9] for a reciprocity formula modulo 8 ).

Rademacher [12] defined

$$
f(d / c)=s(d, c)
$$

as a function on the rationals. The function $f(x)$ is well-defined, since it can be shown from the definition that $s(q d, q c)=s(d, c)$ for any positive integer $q$. Rademacher posed the following questions about $f(x)$. The first was raised in [12], the others in [13, p. 112]:

1) Is $f(x)$ unbounded in any neighborhood of any rational number?

2) Are the points $(x, f(x))$ dense in $\mathbb{R} \times \mathbb{R}$ ?

3) Given two adjacent Farey fractions, $h_{1} / k_{1}$ and $h_{2} / k_{2}$ with $f\left(h_{1} / k_{1}\right)>$ 0 and $f\left(h_{2} / k_{2}\right)>0$, does it follow that

$$
f\left(\frac{h_{1}+h_{2}}{k_{1}+k_{2}}\right) \geq 0 ?
$$


In [12], Rademacher answers the first question in the affirmative. D. Hickerson [8] answers the second, also in the affirmative, using a continued fraction representation for $s(d, c)$. Several authors [1], [11], [14] have answered the third in the negative. All of these authors make extensive use of (1.3) to prove their results. Because of the constant right-hand side of (1.4) and the fact that the parities of $c$ and $d$ are more restricted, one cannot employ the methods of Rademacher and Hickerson. Thus to study the corresponding problems for the sums defined in (1.1) and (1.2) we need to devise new methods. In Section 3, we answer the analogous questions about the sums associated with the theta-functions. The first two results of Section 2 are used extensively in the sequel.

\section{Main results}

Proposition 1. Let $(c, d)=1$ with $c>0$.

(i) If $c$ and $d$ are both odd, then $S(d, c)=0$.

(ii) If $d$ is even, then $S_{4}(d, c)=0$.

Proof. The proof of (i) is presented directly.

Since $d$ and $c$ are odd, $d=c-2 n$ for some $n \in \mathbb{Z}$. Then $(c, n)=1$ since $(c, d)=1$. Therefore, using the definition of $S(d, c)$ and the fact that $[\alpha]+[-\alpha]=-1$ for $\alpha \notin \mathbb{Z}$, we see that

$$
\begin{aligned}
S(d, c)=S(c-2 n, c) & =\sum_{j=1}^{c-1}(-1)^{j+1+[(c-2 n) j / c]} \\
& =\sum_{j=1}^{c-1}(-1)^{2 j+1+[-2 n j / c]}=\sum_{j=1}^{c-1}(-1)^{[2 n j / c]} .
\end{aligned}
$$

Next we show that the sum of the $j$ th and the $(c-j)$ th exponents in (2.1) is odd and thus they have opposite parity. Therefore the corresponding terms cancel each other. Now, since $[\alpha]+[-\alpha]=-1$ for $\alpha \notin \mathbb{Z}$,

$$
\left[\frac{2 n j}{c}\right]+\left[\frac{2 n(c-j)}{c}\right]=\left[\frac{2 n j}{c}\right]+2 n+\left[-\frac{2 n j}{c}\right]=2 n-1 .
$$

There are an even number of terms, so we conclude that $S(d, c)=0$. The deduction of (ii) is made by observing that the exponents have the same form as those in the last sum in (2.1).

Rem ark. Some properties about these analogous sums, including Proposition 1, are proved by R. Sitaramachandrarao in [15].

TheOREM 2. Let $(a, b)=1$ with $0 \leq a \leq b$, and let $x, y$ be nonnegative integers such that ay $-b x= \pm 1$ and $y>0$. Then for $k \in \mathbb{N}$, 
(i) $S(x+a k, y+b k)=\sum_{l=0}^{k-1}(-1)^{(a+b) l}\left\{S(a, b) \pm(-1)^{a+b}\right\}+(-1)^{(a+b) k} S(x, y)$

and

(ii) $S_{4}(x+a k, y+b k)=\sum_{l=0}^{k-1}(-1)^{a l}\left\{S_{4}(a, b) \mp(-1)^{a}\right\}+(-1)^{a k} S_{4}(x, y)$.

The following lemmas will be used to prove Theorem 2 .

Lemma 3. Let $(a, b)=1$ with $0 \leq a \leq b$. Let $x, y, m, l, k$ be nonnegative integers such that ay $-b x= \pm 1, y>0,1 \leq m \leq b$, and $0 \leq l<k$. Then

$$
\left[\frac{(x+a k) m \mp l}{y+b k}\right]=\left[\frac{(x+a k) m}{y+b k}\right] .
$$

If, in addition, $m<y$, then

$$
\left[\frac{(x+a k) m \mp k}{y+b k}\right]=\left[\frac{(x+a k) m}{y+b k}\right] .
$$

Pr o of. For $l=0$, there is nothing to prove. For $1 \leq l<k$, it is sufficient to show that

Suppose that

$$
\left[\frac{(x+a k) m \mp(k-1)}{y+b k}\right]=\left[\frac{(x+a k) m}{y+b k}\right] .
$$

$$
\left[\frac{(x+a k) m \mp(k-1)}{y+b k}\right] \neq\left[\frac{(x+a k) m}{y+b k}\right] .
$$

Then, since $b \geq 1, y \geq 1$ and consequently $k-1<y+b k$, we have

$$
\left[\frac{(x+a k) m \mp(k-1)}{y+b k}\right]=\left[\frac{(x+a k) m}{y+b k}\right] \mp 1 .
$$

If (2.3) holds, then there is an integer $j$ with $1 \leq j \leq k-1$ such that

$$
y+b k \mid(x+a k) m \mp j .
$$

We show that (2.4) is impossible, so the assumption (2.2) is false.

If (2.4) holds, then there exists an integer $t>0$ such that

$$
(y+b k) t=(x+a k) m \mp j .
$$

Now

$$
a(y+b k)-b(x+a k)= \pm 1,
$$

so we also have

$$
-a j(y+b k)-(-b j)(x+a k)=\mp j .
$$

And from [10, p. 213], any solution of the Diophantine equation

$$
A(y+b k)-B(x+a k)=\mp j
$$


has the form

$$
A=-a j+n(x+a k), \quad B=-b j+n(y+b k), \quad n \in \mathbb{Z},
$$

Thus from (2.5), (2.7), and (2.8), for a particular $n_{0} \in \mathbb{Z}$, we have

$$
m=-b j+n_{0}(y+b k) \text { and } t=-a j+n_{0}(x+a k) .
$$

We rearrange the expression for $m$ to get

$$
m=b\left(n_{0} k-j\right)+n_{0} y .
$$

If $n_{0}>0$, then from (2.9), since $j<k$, we are forced to conclude $m>b$. If $n_{0} \leq 0$, then we must have $m<0$. In any case, since $1 \leq m \leq b$ by hypothesis, we have a contradiction to (2.4).

If $m<y$, similar arguments rule out all values for $n_{0}$ when $j=k$, and we make the second conclusion in the statement of the lemma.

Lemma 4. Let $a, b, m, x, y$ be nonnegative integers with $(a, b)=1,1 \leq$ $m<b$, ay $-b x= \pm 1$, and $y>0$. Then for $k \in \mathbb{N}$,

$$
\left[\frac{(x+a k) m}{y+b k}\right]=\left[\frac{a m}{b}\right] \text {. }
$$

Proof. Note that since $(a, b)=1$ and $m<b, b \nmid a m$. Using this fact, $a y-b x= \pm 1$, and the inequality $m /(y+b k)<1$, we conclude that

$$
\begin{aligned}
{\left[\frac{(x+a k) m}{y+b k}\right] } & =\left[\frac{(x+a k) m}{y+b k}-\frac{a m}{b}+\frac{a m}{b}\right]=\left[\frac{a m}{b} \mp \frac{m}{b(y+b k)}\right] \\
& =\left[\frac{a m}{b} \mp \frac{m /(y+b k)}{b}\right]=\left[\frac{a m}{b}\right] .
\end{aligned}
$$

Lemma 5. Let $a, b, x, y, m$ be nonnegative integers with $(a, b)=1$, ay$b x= \pm 1, y>0$, and $1 \leq m<y$. Then for $k \in \mathbb{N} \cup\{0\}$,

$$
\left[\frac{(x+a k) m}{y+b k}\right]=\left[\frac{x m}{y}\right] \text {. }
$$

Proof. We use induction on $k$. When $k=0$,

$$
\left[\frac{(x+a \cdot 0) m}{y+b \cdot 0}\right]=\left[\frac{x m}{y}\right] \text {. }
$$

Assuming (2.10) for $k \geq 1$, we see that, since $a y-b x= \pm 1$,

$$
\begin{aligned}
{\left[\frac{x m}{y}\right] } & =\left[\frac{(x+a k) m}{y+b k}\right] \\
& =\left[\frac{(x+a k) m}{y+b k}+\frac{m(x+a(k+1))}{y+b(k+1)}-\frac{m(x+a(k+1))}{y+b(k+1)}\right]
\end{aligned}
$$




$$
\begin{aligned}
& =\left[\frac{m(x+a(k+1))}{y+b(k+1)} \mp \frac{m}{(y+b k)(y+b(k+1))}\right] \\
& =\left[\frac{m(x+a(k+1))}{y+b(k+1)} \mp \frac{m /(y+b k)}{y+b(k+1)}\right] .
\end{aligned}
$$

Now since $m /(y+b k)<1$ and, from (2.6), $y+b(k+1) \nmid m(x+a(k+1))$,

$$
\left[\frac{x m}{y}\right]=\left[\frac{m(x+a(k+1))}{y+b(k+1)}\right] .
$$

Proof of Theorem 2. We prove (i). The proof of (ii) follows the same steps.

From (1.1),

$$
S(x+a k, y+b k)=\sum_{j=1}^{y+b k-1}(-1)^{j+1+\left[\frac{(x+a k) j}{y+b k}\right]}=R_{1}+R_{2},
$$

where

$$
R_{1}:=\sum_{j=1}^{b k}(-1)^{j+1+\left[\frac{(x+a k) j}{y+b k}\right]}
$$

and

$$
R_{2}:=\sum_{j=b k+1}^{b k+y-1}(-1)^{j+1+\left[\frac{(x+a k) j}{y+b k}\right]} .
$$

First,

$$
\begin{aligned}
R_{1} & =\sum_{l=0}^{k-1} \sum_{j=b l+1}^{b(l+1)}(-1)^{j+1+\left[\frac{(x+a k) j}{y+b k}\right]} \\
& =\sum_{l=0}^{k-1} \sum_{m=1}^{b}(-1)^{m+b l+1+\left[\frac{(x+a k)(m+b l)}{y+b k}\right]} .
\end{aligned}
$$

Now since $a y-b x= \pm 1$,

$$
\begin{aligned}
{\left[\frac{(x+a k)(m+b l)}{y+b k}\right] } & =\left[\frac{(x+a k) m+x b l+a b k l}{y+b k}\right] \\
& =\left[\frac{(x+a k) m+(a y \mp 1) l+a b k l}{y+b k}\right] \\
& =\left[\frac{(x+a k) m \mp l+a l(y+b k)}{y+b k}\right] \\
& =\left[\frac{(x+a k) m \mp l}{y+b k}\right]+a l .
\end{aligned}
$$

So from (2.12), (2.13), and Lemma 3(i), 


$$
R_{1}=\sum_{l=0}^{k-1}(-1)^{(a+b) l} \sum_{m=1}^{b}(-1)^{m+1+\left[\frac{(x+a k) m}{y+b k}\right]} .
$$

Now, separating the inner sum and then applying Lemma 4, we find that

$$
R_{1}=\sum_{l=0}^{k-1}(-1)^{(a+b) l}\left\{\sum_{m=1}^{b-1}(-1)^{m+1+\left[\frac{a m}{b}\right]}+(-1)^{b+1+\left[\frac{(x+a k) b}{y+b k}\right]}\right\} .
$$

Simplifying the exponent of the last term inside the braces in (2.14), we see that

$$
\begin{aligned}
b+1+\left[\frac{(x+a k) b}{y+b k}\right] & =b+1+\left[\frac{b x+a k b}{y+b k}\right] \\
& =b+1+\left[\frac{a y \mp 1+a k b}{y+b k}\right] \\
& =b+1+a+\left[\frac{\mp 1}{y+b k}\right] .
\end{aligned}
$$

Then we have, from $(2.14),(2.15)$ and the definition of $S(a, b)$,

$$
R_{1}=\sum_{l=0}^{k-1}(-1)^{(a+b) l}\left\{S(a, b) \pm(-1)^{a+b}\right\} .
$$

To evaluate $R_{2}$, we use $a y-b x= \pm 1$, Lemma 3(ii), Lemma 5 , and the definition of $S(x, y)$ to deduce that

$$
\begin{aligned}
R_{2} & =\sum_{j=b k+1}^{b k+y-1}(-1)^{j+1+\left[\frac{(x+a k) j}{y+b k}\right]} \\
& =\sum_{m=1}^{y-1}(-1)^{m+b k+1+\left[\frac{(x+a k)(m+b k)}{y+b k}\right]} \\
& =\sum_{m=1}^{y-1}(-1)^{m+b k+1+a k+\left[\frac{(x+a k) m \mp k}{y+b k}\right]} \\
& =(-1)^{(a+b) k} \sum_{m=1}^{y-1}(-1)^{m+1+\left[\frac{(x+a k) m}{y+b k}\right]} \\
& =(-1)^{(a+b) k} \sum_{m=1}^{y-1}(-1)^{m+1+\left[\frac{x m}{y}\right]}=(-1)^{(a+b) k} S(x, y) .
\end{aligned}
$$

Upon combining (2.11), (2.16), and (2.17), we reach the conclusion $S(x+a k, y+b k)=\sum_{l=0}^{k-1}(-1)^{(a+b) l}\left\{S(a, b) \pm(-1)^{a+b}\right\}+(-1)^{(a+b) k} S(x, y)$. 
COROLlary 6. Let $(a, b)=1$ with $0 \leq a \leq b$, and let $x, y$ be nonnegative integers such that $a y-b x= \pm 1$ and $y>0$.

(i) If $a$ and $b$ are both odd, then

$$
S(x+a k, y+b k)= \pm k+S(x, y) .
$$

(ii) If a is even, then

$$
S_{4}(x+a k, y+b k)=\mp k+S_{4}(x, y) .
$$

Proof. To prove (i), we use Theorem 2(i), the fact that $a+b$ is even, and Proposition 1(i). The proof of (ii) is similar.

Corollary 7. Let $(a, b)=1$ with $0 \leq a \leq b$, and let $x, y$ be nonnegative integers such that $a y-b x= \pm 1$ and $y>0$.

(i) If $a+b$ is odd and $x$ and $y$ are both odd, then

$$
S(x+a k, y+b k)= \begin{cases}0 & \text { if } k \text { is even }, \\ S(a, b) \mp 1 & \text { if } k \text { is odd. }\end{cases}
$$

(ii) If $a$ is odd and $x$ is even, then

$$
S_{4}(x+a k, y+b k)= \begin{cases}0 & \text { if } k \text { is even }, \\ S_{4}(a, b) \pm 1 & \text { if } k \text { is odd. }\end{cases}
$$

Proof. To prove (i), we note that, from Theorem 2(i) and Proposition $1(\mathrm{i})$,

$$
\begin{aligned}
S(x+a k, y+b k) & =\sum_{l=0}^{k-1}(-1)^{l}\{S(a, b) \mp 1\} \\
& = \begin{cases}0 & \text { if } k \text { is even, } \\
S(a, b) \mp 1 & \text { if } k \text { is odd. }\end{cases}
\end{aligned}
$$

The proof of (ii) is similar.

Remark. In the cases when $k$ is even in Corollary 7 the sums on the left are equal to zero by Proposition 1.

Corollary 8. Let $(a, b)=1$ with $0 \leq a \leq b$, and let $x, y$ be nonnegative integers such that $a y-b x= \pm 1$ and $y>0$.

(i) If $a+b$ is odd and $x+y$ is odd, then

$$
S(x+a k, y+b k)= \begin{cases}S(a, b) \mp 1 & \text { if } k \text { is even }, \\ 0 & \text { if } k \text { is odd. }\end{cases}
$$

(ii) If $a$ is odd and $x$ is odd, then

$$
S_{4}(x+a k, y+b k)= \begin{cases}S_{4}(a, b) \pm 1 & \text { if } k \text { is even } \\ 0 & \text { if } k \text { is odd. }\end{cases}
$$


Proof. We prove (i). The proof of (ii) follows the same steps. If $k=1$, recall that $a y-b x= \pm 1$, so that, because of the parities of $a, b, x$, and $y$, the result follows from Proposition 1(i). If $k \geq 2$, (i) follows from Theorem 2(i) and Proposition 1(i) since

$$
\begin{aligned}
S(x+a k, y+b k) & =S(x+a+a(k-1), y+b+b(k-1)) \\
& =\sum_{l=0}^{k-2}(-1)^{l}\{S(a, b) \mp 1\}+(-1)^{k-1} S(x+a, y+b) \\
& =\sum_{l=0}^{k-2}(-1)^{l}\{S(a, b) \mp 1\} \\
& = \begin{cases}S(a, b) \mp 1 & \text { if } k \text { is even, } \\
0 & \text { if } k \text { is odd. }\end{cases}
\end{aligned}
$$

Corollary 9. Let $(a, b)=1$ with $0 \leq a \leq b$, and let $x, y$ be nonnegative integers such that $a y-b x= \pm 1$ and $y>0$.

(i) If $a+b$ is odd and $x+y$ is odd, then

$$
S(a, b)-S(x, y)= \pm 1 \text {. }
$$

(ii) If $a$ is odd and $x$ is odd, then

$$
S_{4}(a, b)-S_{4}(x, y)=\mp 1 .
$$

Proof. We apply Theorem 2(i) with $k=1$ to get

$$
S(x+a, y+b)=S(a, b) \pm(-1)^{a+b}+(-1)^{a+b} S(x, y) .
$$

By Corollary 8(i), with $k=1$,

$$
S(x+a, y+b)=0 .
$$

The result follows from (2.18) and (2.19), since $a+b$ is odd. As usual, (ii) is proved similarly.

Corollary 10. Let $(a, b)=1$ with $0 \leq a \leq b$, and let $x, y$ be nonnegative integers such that ay $-b x= \pm 1$ and $y>0$.

(i) If $a+b$ is odd, $x+y$ is odd and $k$ is even, then

$$
S(x+a k, y+b k)=S(x, y) .
$$

(ii) If $a$ is odd, $x$ is odd and $k$ is even, then

$$
S_{4}(x+a k, y+b k)=S_{4}(x, y) .
$$

Pro of. To prove (i), we have, from Corollary 8(i),

$$
S(x+a k, y+b k)=S(a, b) \mp 1 .
$$

Then the result follows immediately from (2.20) and Corollary 9(i). The proof of (ii) is similar. 
3. Rademacher's questions. We are now in a position to address the questions of Rademacher as they relate to the sum $S(d, c)$. Let $(d, c)=1$ and define $g(d / c)=S(d, c)$. Here we require that $c$ and $d$ be relatively prime, since $S(q d, q c) \neq S(d, c)$ in general. The analogues of Rademacher's questions are:

1) Is $g(x)$ unbounded in any neighborhood of any real number?

2) Are the points $(x, g(x))$ dense in $\mathbb{R} \times \mathbb{Z}$ ?

3) Given two adjacent Farey fractions, $h_{1} / k_{1}$ and $h_{2} / k_{2}$ with $g\left(h_{1} / k_{1}\right)>$ 0 and $g\left(h_{2} / k_{2}\right)>0$, does it follow that

$$
g\left(\frac{h_{1}+h_{2}}{k_{1}+k_{2}}\right) \geq 0 ?
$$

Note that $g(x)$ is periodic with period 2 , since

$$
\begin{aligned}
g\left(\frac{d}{c}+2\right) & =S(d+2 c, c)=\sum_{j=1}^{c-1}(-1)^{j+1+[(d+2 c) j / c]} \\
& =\sum_{j=1}^{c-1}(-1)^{3 j+1+[d j / c]}=\sum_{j=1}^{c-1}(-1)^{j+1+[d j / c]} \\
& =S(d, c)=g\left(\frac{d}{c}\right) .
\end{aligned}
$$

Also observe that

$$
\begin{aligned}
S(d+c, c) & =\sum_{j=1}^{c-1}(-1)^{j+1+[(d+c) j / c]}=\sum_{j=1}^{c-1}(-1)^{2 j+1+[d j / c]} \\
& =-\sum_{j=1}^{c-1}(-1)^{[d j / c]}=-S_{4}(d, c) .
\end{aligned}
$$

We now show that, as in the case of Dedekind sums, Question 1 can be answered affirmatively.

THEOREM 11. The function $g(x)$ is unbounded above and below in any neighborhood of any real number.

Proof. Let $r \in \mathbb{R}$ and $\varepsilon>0$ be given. Because of (3.1), we may assume that $r \in[0,2)$. If $r \in[0,1]$, then there exists $a / b$ such that $(a, b)=1, a$ and $b$ are both odd, and

$$
\left|r-\frac{a}{b}\right|<\varepsilon .
$$

Let $x_{1}, y_{1} \in \mathbb{N}$ be such that $a y_{1}-b x_{1}=1$. Then for large enough $k \in \mathbb{N}$,

$$
\left|r-\frac{x_{1}+a k}{y_{1}+b k}\right|<\varepsilon .
$$


Now, applying Corollary 6(i), we see that

$$
g\left(\frac{x_{1}+a k}{y_{1}+b k}\right)=k+O(1) \quad \text { as } k \rightarrow \infty .
$$

Similarly, there exist $x_{2}, y_{2} \in \mathbb{N}$ such that $a y_{2}-b x_{2}=-1$ and

$$
\left|r-\frac{x_{2}+a k}{y_{2}+b k}\right|<\varepsilon
$$

Then, by Corollary 6(i) again, we have

$$
g\left(\frac{x_{2}+a k}{y_{2}+b k}\right)=-k+O(1) \quad \text { as } k \rightarrow \infty .
$$

Thus, from (3.3) and (3.4), $g(x)$ is arbitrarily large in either direction in this neighborhood.

If $r \in(1,2)$, we apply a similar argument to $r-1$. There exists $a / b$ with $(a, b)=1, a$ even, and

$$
\left|r-1-\frac{a}{b}\right|<\varepsilon
$$

Now let $x_{1}, y_{1} \in \mathbb{N}$ with $a y_{1}-b x_{1}=1$. Then for large enough $k \in \mathbb{N}$,

$$
\left|r-1-\frac{x_{1}+a k}{y_{1}+b k}\right|<\varepsilon .
$$

Then by Corollary 6(ii),

$$
S_{4}\left(x_{1}+a k, y_{1}+b k\right)=-k+S_{4}\left(x_{1}, y_{1}\right) .
$$

Thus from (3.2) and (3.6), we get

$$
S\left(x_{1}+a k+y_{1}+b k, y_{1}+b k\right)=-S_{4}\left(x_{1}+a k, y_{1}+b k\right)=k-S_{4}\left(x_{1}, y_{1}\right) \text {. }
$$

In other words,

$$
g\left(\frac{x_{1}+a k+y_{1}+b k}{y_{1}+b k}\right)=k+O(1) \quad \text { as } k \rightarrow \infty .
$$

And from (3.5),

$$
\left|r-\frac{x_{1}+a k+y_{1}+b k}{y_{1}+b k}\right|<\varepsilon .
$$

So we conclude from (3.7) that $g(x)$ is unbounded from above for $x \in(1,2)$. An identical argument shows that $g(x)$ is also unbounded from below on $(1,2)$.

We now answer Question 2. Again, as with Dedekind sums, we answer in the affirmative.

TheOREM 12. The points $(x, g(x))$ are dense in $\mathbb{R} \times \mathbb{Z}$.

The proof of Theorem 12 depends upon two additional results. The first, due to Goldberg [5, pp. 109-110], is 
Theorem 13. Let $(c, d)=1$ with $d^{2} \equiv-1(\bmod c)$.

(i) If $c$ is odd and $d$ is even, then $S(d, c)=0$.

(ii) If $c$ and $d$ are both odd, then $S_{4}(d, c)=0$.

The second is due to W. Duke, J. B. Friedlander, and H. Iwaniec [4]. Let $f(x)$ be an irreducible quadratic polynomial with integral coefficients and negative discriminant and let $p$ be prime. Let $p$ vary and take all numbers of the form $\nu / p$, where $f(\nu) \equiv 0(\bmod p)$ and $0<\nu \leq p$. Arrange the numbers $\nu / p$ as a sequence $n_{1}, \ldots, n_{m}, \ldots$, so that the corresponding denominators $p$ are in ascending order.

TheOREM 14. The sequence $n_{1}, \ldots, n_{m}, \ldots$, as defined above, is uniformly distributed in the interval $(0,1)$.

Proof of Theorem 12. It is sufficient to show that the function $g(x)$ takes on every integer value in any neighborhood of a number $r$. Also, by (3.1), we need only consider $r \in[0,2)$.

Let $\varepsilon>0, r \in[0,1]$, and our desired value $M \in \mathbb{Z}$ be given.

We want to find $a_{0} / b_{0}$ such that $\left(a_{0}, b_{0}\right)=1, b_{0}$ is odd, $a_{0}$ is even, $a_{0}^{2} \equiv-1\left(\bmod b_{0}\right)$, and

$$
\left|r-\frac{a_{0}}{b_{0}}\right|<\varepsilon
$$

This can be accomplished by applying Theorem 14 in a way that will guarantee that $a_{0}$ is even. First let $f(x)=4 x^{2}+1$. Theorem 14 asserts that there is a $\nu$ and an odd prime $p$ such that $4 \nu^{2} \equiv-1(\bmod p)$ and

$$
\left|\frac{r}{2}-\frac{\nu}{p}\right|<\frac{\varepsilon}{2} \text {. }
$$

Now set $a_{0}=2 \nu$ and $b_{0}=p$ and the conditions are satisfied.

If $M=0$ we stop since, by Theorem 13(i), $g\left(a_{0} / b_{0}\right)=0$. And we have found a number in the neighborhood giving the desired value. Suppose $M>0$. Let $x_{0}, y_{0}>0$ be such that $a_{0} y_{0}-b_{0} x_{0}=-1$ and $x_{0}+y_{0}$ is odd. Then from (3.8), for sufficiently large $k$,

$$
\left|r-\frac{x_{0}+a_{0} k}{y_{0}+b_{0} k}\right|<\varepsilon
$$

Now by Corollary 8(i) and Theorem 13(i), for some large even $k_{0}$,

$$
g\left(\frac{x_{0}+a_{0} k_{0}}{y_{0}+b_{0} k_{0}}\right)=S\left(x_{0}+a_{0} k_{0}, y_{0}+b_{0} k_{0}\right)=S\left(a_{0}, b_{0}\right)+1=1 .
$$

If $M=1$ we stop, since we have found a number in the neighborhood giving the value $M$. If $M>1$, set

$$
a_{1}=x_{0}+a_{0} k_{0} \quad \text { and } \quad b_{1}=y_{0}+b_{0} k_{0} .
$$


Since $\left(a_{1}, b_{1}\right)=1$, there exist $x_{1}, y_{1}>0$ such that $a_{1} y_{1}-b_{1} x_{1}=-1$ and $x_{1}+y_{1}$ is odd. Then from (3.8), for sufficiently large $k$,

$$
\left|r-\frac{x_{1}+a_{1} k}{y_{1}+b_{1} k}\right|<\varepsilon
$$

From Corollary 8(i) and (3.9), with a sufficiently large even $k_{1}$, we deduce that

$$
g\left(\frac{x_{1}+a_{1} k_{1}}{y_{1}+b_{1} k_{1}}\right)=g\left(\frac{a_{1}}{b_{1}}\right)+1=2 .
$$

We continue this process $M$ times to find $x_{M-1}, y_{M-1}$ and $a_{M-1}, b_{M-1}$ such that $x_{M-1}+y_{M-1}$ and $a_{M-1}+b_{M-1}$ are odd, $a_{M-1} y_{M-1}-b_{M-1} x_{M-1}$ $=-1$, and

$$
S\left(a_{M-1}, b_{M-1}\right)=M-1,
$$

and for large enough $k$,

$$
\left|r-\frac{x_{M-1}+a_{M-1} k}{y_{M-1}+b_{M-1} k}\right|<\varepsilon
$$

Choosing a large enough even $k_{M-1}$ and using Corollary $8(\mathrm{i})$, we find that

$$
g\left(\frac{x_{M-1}+a_{M-1} k_{M-1}}{y_{M-1}+b_{M-1} k_{M-1}}\right)=g\left(\frac{a_{M-1}}{b_{M-1}}\right)+1=M-1+1=M .
$$

If $M<0$, then we apply the same argument, except that we find appropriate $a, b, x, y$ with $a y-b x=1$.

If $r \in(1,2)$, we apply the argument used in the proof of Theorem 11, using Corollary 8(ii), Theorem 13(ii) and finally (3.2).

We now address Question 3. Here, unlike the situation with Dedekind sums, the question, as posed, has a simple answer. Suppose that $h_{1} / k_{1}$ and $h_{2} / k_{2}$ are adjacent Farey fractions with $g\left(h_{1} / k_{1}\right), g\left(h_{2} / k_{2}\right) \neq 0$. Recall that, from elementary properties of Farey fractions, $h_{1} k_{2}-h_{2} k_{1}= \pm 1$. Then by Proposition 1(i), $h_{1}+k_{1}$ and $h_{2}+k_{2}$ are odd. Thus $h_{1}+h_{2}$ and $k_{1}+k_{2}$ are both odd and by Proposition 1(i),

$$
g\left(\frac{h_{1}+h_{2}}{k_{1}+k_{2}}\right)=S\left(h_{1}+h_{2}, k_{1}+k_{2}\right)=0
$$

From Corollary 9(i), we deduce a more interesting result.

THEOREM 15. Let $h_{1} / k_{1}<h_{2} / k_{2}$ be adjacent Farey fractions. If $g\left(h_{1} / k_{1}\right)$ $>($ resp. $<)$, then

$$
g\left(\frac{h_{2}}{k_{2}}\right) \geq(\text { resp. } \leq) 0 .
$$

Proof. If $h_{2}$ and $k_{2}$ are both odd, then, by Proposition 1(i), $g\left(h_{2} / k_{2}\right)$ $=0$. 
If $h_{2}+k_{2}$ is odd, then, by Corollary $9(\mathrm{i})$,

$$
S\left(h_{2}, k_{2}\right)=1+S\left(h_{1}, k_{1}\right)>(\text { resp. } \leq) 0 .
$$

Identical arguments can be used to show that Theorems 11, 12, and 15 hold for the function $g_{4}(x)$, defined by $g_{4}(d / c)=S_{4}(d, c)$ with $(d, c)=1$.

\section{References}

[1] T. Asai, Some arithmetic on Dedekind sums, J. Math. Soc. Japan 38 (1986), $163-172$.

[2] B. C. Berndt, Analytic Eisenstein series, theta-functions, and series relations in the spirit of Ramanujan, J. Reine Angew. Math. 303/304 (1978), 332-365.

[3] R. Dedekind, Erläuterungen zu zwei Fragmenten von Riemann, in: Riemann's Gesammelte Math. Werke, 2nd ed., 1882, 466-472.

[4] W. Duke, J. B. Friedlander and H. Iwaniec, Equidistribution of roots of a quadratic congruence to prime moduli, Ann. of Math. 141 (1995), 423-441.

[5] L. A. Goldberg, Transformations of theta-functions and analogues of Dedekind sums, thesis, Univ. of Illinois, Urbana, 1981.

[6] G. H. Hardy, On certain series of discontinuous functions connected with the modular functions, Quart. J. Math. Oxford 36 (1905), 93-123.

[7] —, Collected Papers, Vol. IV, Clarendon Press, Oxford, 1969.

[8] D. Hickerson, Continued fractions and density results for Dedekind sums, J. Reine Angew. Math. 290 (1977), 113-116.

[9] J. L. Meyer, Analogues of Dedekind sums, thesis, Univ. of Illinois, Urbana, 1997.

[10] I. Niven, H. Zuckerman and H. Montgomery, An Introduction to the Theory of Numbers, 5th ed., Wiley, 1991.

[11] L. Pinzur, On a question of Rademacher concerning Dedekind sums, Proc. Amer. Math. Soc. 61 (1976), 11-15.

[12] H. Rademacher, Zur Theorie der Dedekindschen Summen, Math. Z. 63 (1956), 445-463.

[13] - Questions 99 and 100, in: Proc. 1963 Number Theory Conference, Univ. of Colorado, Boulder, Colo., 1963, 112.

[14] K. Rosen, On the sign of some Dedekind sums, J. Number Theory 9 (1977), 209-212.

[15] R. Sitaramachandrarao, Dedekind and Hardy sums, Acta Arith. 48 (1987), $325-340$.

Department of Mathematics

Syracuse University

Syracuse, New York 13244-1150

U.S.A.

E-mail: jmeyer@math.syr.edu

Received on 9.7.1996

and in revised form on 11.4.1997 